Mitteilungen der Österreichischen Geographischen Gesellschaft, 161. Jg., S. 414-421

(Annals of the Austrian Geographical Society, Vol. 161, pp. 414-421)

Wien (Vienna) 2019, https://doi.org/10.1553/moegg161s414

\title{
Verleihung des Einspieler-Preises an Peter Jordan - MIT EINER LAUDATIO VON VALENTIN INZKO
}

\author{
mit 1 Abb. im Text
}

Am 18. November 2019 wurde HR Prof. h.c. Univ.-Doz. Dr. Peter JoRDAN, langjähriges Vorstandsmitglied der Österreichischen Geographischen Gesellschaft (ÖGG) und Schriftleiter der „Mitteilungen der Österreichischen Geographischen Gesellschaft“ (MÖGG) von 2009 bis 2017 sowie ehemaliger Vorsitzender und jetziger Ehrenvorsitzender der „Arbeitsgemeinschaft für Kartographische Ortsnamenkunde“ (AKO) in Klagenfurt in feierlichem Rahmen der „Einspieler-Preis“ verliehen - als Dank für sein Bemühen um die Anerkennung und Bewahrung geographischer Namen ethnischer Minderheiten, was zur Stärkung der räumlichen Identität und Kultur zwei- und mehrsprachiger Gebiete beiträgt.

Peter JoRDAN ist der erste Geograph, der diese bedeutende Auszeichnung erhielt. Der Einspieler-Preis wird jenen deutschsprachigen Persönlichkeiten aus dem öffentlichen Leben und der Wissenschaft verliehen, die die Anliegen der slowenischen Volksgruppe unterstützen und zum guten Zusammenleben beider Volksgruppen in Kärnten beitragen. Er wurde 1988 vom „Rat der Kärntner Slowenen“ und dem „Christlichen Kulturverband“ gestiftet.

Der Preis ist nach Msgr. Prof. Andrej EINSPIELER benannt. Dieser war Geistlicher, Politiker, Publizist und Volksbildner. Er setzte sich für die Gleichberechtigung der slowenischen Sprache und der Slowenen, für das Zusammenleben beider Volksgruppen in Kärnten, das allgemeine Wahlrecht und die politische Freiheit ein. Als führende politische Persönlichkeit der Kärntner Slowenen war er Abgeordneter zum Kärntner Landtag in den Jahren 1862 bis 1863, 1871 bis 1878 und 1880 bis 1888.

Die Preisverleihung an Peter JoRDan, Kulturgeograph, Kartograph und Ortsnamenkundler (Toponomast), wurde in Kärnten und in Slowenien medial viel beachtet, da er sich in seiner wissenschaftlichen Arbeit immer auch für die Sichtbarmachung und Bewahrung der slowenischen geographischen Namen in Kärnten eingesetzt hat. Die Laudatio bei der Festveranstaltung hielt Dr. Valentin InzKo, Vorsitzender des Rates der Kärntner Slowenen und Hoher Repräsentant der Vereinten Nationen für Bosnien und Herzegowina. Sie und die Dankesworte des Geehrten werden im Folgenden abgedruckt.

Glückwünsche und Grußworte, die bei der Preisverleihung vorgetragen wurden, übermittelten u. a. auch der Vorsitzende der AKO, Dr. Gerhard RAMPL, und der Vorsitzende der Regierungskommission für die Standardisierung geographischer Namen in der Republik Slowenien, Dr. Matjaž GERŠIČ. Peter JORDAN gestand in seiner Dankesrede seine „Rührung über die übergroße Dankbarkeit, die ihm mit diesem Preis entgegengebracht wird für etwas, das jeder andere anständige Mensch in meiner Position und mit meinen Möglichkeiten wohl auch getan hätte - sich für Minderheitenrechte einzusetzen, eigentlich auch nur für die längst fällige Durchsetzung bereits verbriefter Minderheitenrechte“.

Auch die Schriftleitung der MÖGG gratuliert Peter JorDan sehr herzlich zu dieser Auszeichnung, zusätzlich auch zu seinem 70. Geburtstag, der ebenfalls im Jahr 2019 zu feiern war.

Die Schriftleitung ${ }^{1)}$

\footnotetext{
1) Als Schriftleiter der MÖGG danke ich Frau Mag. Martina Pıко-RUSTIA (Slowenisches Volkskundeinstitut Urban Jarnik, Klagenfurt) sehr herzlich für ihre wertvolle Unterstützung und Koordinationstätigkeit für das Zustandekommen dieses Berichts und für die Bereitstellung wichtiger Informationen über den Einspieler-Preis und die Festveranstaltung.
} 


\title{
ANSPRACHE Von Dr. VALENTIN INZKo ${ }^{2)}$
}

\author{
Vorsitzender des Rates der Kärntner Slowenen \\ Hoher Repräsentant der Vereinten Nationen für Bosnien und Herzegowina
}

Verehrter Herr Professor Jordan, liebe Anwesende! Dragi prijatelji! Liebe Freunde! () $^{3}$

Neben der persönlichen Überzeugung, den Werten und der Weltanschauung sind im Leben vor allem Familie, Freunde und gute Nachbarn von Bedeutung. Ein solcher Freund ist Professor Peter Jordan, der ohne jedwede Anregung der Minderheit, als Nicht-Slowene, die slowenischen topographischen Aufschriften zu erforschen begann. Noch bedeutsamer aber ist, dass er schon im Jahr 1988 erstmals über die Notwendigkeit der Einbeziehung von slowenischen Ortsnamen auf amtlichen österreichischen Landkarten schrieb und dies später des Öfteren wiederholte. Das Büchlein über diese Problematik $k^{4)}$ brachte er in das Österreichische Volksgruppenzentrum, wo es mit Freude aufgenommen und dem Parlamentsabgeordneten Karel Smolle überreicht wurde. Diese Frage brachte dann Karel Smolle in die Parlamentsdebatte ein. Die Minderheit hatte damals zwar nur beschämende 91 anerkannte zweisprachige Ortstafeln, dennoch war es Professor Jordans Anliegen, diese Namen in amtliche österreichische Landkarten einzuführen. Heute, da wir alle die Navigation oder das GPS benutzen, frage ich mich am Rande, ob es nicht sinnvoll wäre, die bestehenden zweisprachigen Benennungen auch in diese Systeme einzuführen.

Demnach hat Professor Jordan immense Verdienste, dass das Slowenische auch in der amtlichen österreichischen Kartographie sichtbar wurde. Und das ist nicht wenig. DIE MINDERHEIT WIRD DADURCH SICHTBAR!

Einst sagte mir Milka Kriegl: Ich will nicht nach ACHOMITZ nach Hause kommen, ich möchte nach ZAHOMC nach Hause kommen. Gerade das behauptet auch Professor Jordan. Dass der Heimatort auch Teil der Identität sei, dass mit der Ortstafelaufschrift die Minderheit auch sichtbar sei und dass dies unser gemeinsames Kulturerbe sei - jetzt auch bekannt als immaterielles Kulturerbe, das im diesbezüglichen Verzeichnis der Österreichischen UNESCO-Kommission gelistet ist.

Sehr geehrter Herr Professor!

Wie immer versuche ich am Anfang, einen Bezug zum Namensgeber dieser Auszeichnung herzustellen, obwohl Andrej EINSPIELER bereits vor mehr als hundert Jahren gestorben ist. Nicht wirklich wichtig, aber ein netter Zufall ist da einmal das Geburtsdatum. EINSPIELER wurde am 13. November geboren, Professor JoRDAN am 15. November, vor genau 70 Jahren! Alles Gute!

Beide waren Workaholics, wobei dies zu EInSPIELERs Zeiten wahrscheinlich anders hieß, etwa Arbeitstier oder Arbeitswütiger - mit unbändiger Beharrlichkeit, einem Durchhaltewillen und manchmal auch mit Starrsinn. Wie anders kann man sich erklären, dass der langjährige Landtagsabgeordnete, Professor und Priester, Andrej EinSPIELER, zum Beispiel neun Zeitungen gründete, fünf

2) Die Schriftleitung dankt Herrn Dr. Valentin Inzko sehr herzlich für die Bereitstellung seines Redemanuskripts und die Zustimmung zur Veröffentlichung in den MÖGG.

3) Valentin InZKo hielt den ersten Teil seiner Rede (in Kursivschrift) in slowenischer Sprache. Die Übersetzung erfolgte freundlicherweise durch Martina PIKo-Rustia.

4) JoRDAN Peter (1988): Möglichkeiten einer stärkeren Berücksichtigung slowenischer Ortsnamen in den heutigen amtlichen topographischen Karten Österreichs (= Österreichische Akademie der Wissenschaften, Institut für Kartographie, Berichte und Informationen, 6). Wien: Verlag der ÖAW. 
slowenische und vier deutschsprachige, um den „deutschen Bruder im Lande“ besser ansprechen zu können.

Bei Professor JoRDAn ist es ähnlich. Seinen Lebenslauf und die Liste der Auswahlbibliographie (aus mehr als 400 Publikationen) finden Sie in der Mappe. Ich habe ausgerechnet, dass er in seinem wissenschaftlichen Leben im Schnitt neun bis zehn Publikationen pro Jahr veröffentlichte. Beinahe eine pro Monat! Er leitete zahlreiche Forschungsprojekte, ist Mitherausgeber von zirka 30 wissenschaftlichen Zeitschriften, mehrfaches Ehrenmitglied internationaler Gremien und Akademien und Träger mannigfaltiger Auszeichnungen.

Bei manchen Orten haben wir sogar Berührungspunkte, denn auch ich bin oft in Olmütz, Olomouc in Mähren gewesen, wo er Mitherausgeber einer Publikation war und ist. Auch in Belgrad haben wir beide gewirkt, aber obwohl wir im gleichen Jahr geboren wurden, trafen wir dort einander nie, erst später in Klagenfurt. Eine faszinierende gemeinsame Stätte war auch Prag, wo Professor JoRDAN Ehrenmitglied der Tschechischen Geographischen Gesellschaft ist. Ein weiterer gemeinsamer Ort ist Ljubljana, wo JoRDAN Mitherausgeber des „Geografski zbornik“ ist, auch von „Acta Geographica Slovenica“. Auch der legendäre Professor PlaschKa zählt zu unseren gemeinsamen Bekannten. Ich mache hier Schluss mit den Aufzählungen, denn laut Anleitungen für eine gute Lobesrede darf eine Laudatio nicht zu lange dauern.

Was ist also der Kern seiner Tätigkeit aus der Sicht der Volksgruppe? Ich meine, dass er den für uns interessanten Teil selbst, sehr treffend, so beschrieben hat:

Die stärkere Berücksichtigung slowenischer Ortsnamen in den heutigen amtlichen topographischen Karten Österreichs.

Das bedeutet, Professor JoRDan sucht sein ganzes Leben nach Möglichkeiten, slowenischen Ortsnamen in den amtlichen Karten mehr Geltung zu verschaffen. Das ist doch wunderbar und verdient einen kräftigen Applaus!

Denn die Toponyme oder Ortsnamen sind erstrangige Bestandteile des Kulturgutes eines Volkes, einer Region und eines Staates. Sie müssen laut JoRDAN dokumentiert und publiziert werden, um sie in praktischer Verwendung zu halten und vor dem Vergessen zu schützen. Der Staat, dem der Schutz einer Minderheit anvertraut ist, hat in dieser Hinsicht besondere Verantwortung und als Herausgeber amtlicher Kartenwerke auch besondere Möglichkeiten. Ortsnamen unterstützen laut Professor JoRDAN bei den Angehörigen einer Minderheit außerdem das Gefühl der Verbundenheit mit dem Staat und mit dem Mehrheitsvolk, mit dem sie zusammenleben, wenn sie ihre Heimat in den amtlichen topographischen Karten im Kleid der ihnen vertrauten Namen wiederfinden. Wie jede soziale Gruppe hat auch eine Minderheit - sie vielleicht sogar in stärkerem Maße - das Bedürfnis, ihr Dasein zu manifestieren, zu zeigen: „Hier sind wir.“

Minderheitentoponyme in amtlichen topographischen Karten sind laut JORDAN ein Mittel, diesem Bedürfnis zu entsprechen. Es geht bei der Frage der Berücksichtigung von Minderheitentoponymen in amtlichen Karten aber nicht allein darum, Minderheiten aus humanitären, politischen oder anderen Gründen entgegenzukommen. Es geht laut JoRDAN auch um die topographische Karte selbst, um ihren Wert als umfassendes und ausgewogenes Informationsmittel. Topographische Karten, die auch topographische Bezeichnungen der Minderheit erhalten, leisten mehr für die allgemeine Orientierung, indem sie mehr von der Wirklichkeit einfangen; sie sprechen eine Minderheit als Benützergruppe besser an und bilden laut JORDAN verlässliche Grundlagen toponomastischer, siedlungsgeographischer und siedlungsgeschichtlicher, nationalitäten-geschichtlicher und nationalitäten-politischer Forschungen.

Rein beispielhaft, wie akribisch und mit welcher Liebe zum Detail Professor JORDAN arbeitet: er entdeckte in den Gemeinden Eisenkappel/Železna Kapla und Zell/Sele eingedeutschte topographi- 
sche Bezeichnungen, wie Goli Berg oder Breiter Gupf. Ebenfalls aufgefallen ist ihm jedoch auch die positive Reslowenisierung dieser eingedeutschten Bezeichnungen. Also Goli Berg wieder zu Goli vrh oder Breiter Gupf zu Tolsti vrh. Er benützt aber auch sein inneres Mikroskop und entdeckte zum Beispiel, dass Pistotnik einmal mit „k“ geschrieben wird, dann wieder mit „g“, also Pistotnig. Oder Trattnig mit doppeltem „t“ und „g“ am Ende und ein andermal wieder Tratnik, slowenisch. In verschiedenen Epochen der österreichischen Kartographie werden Vulgonamen mit „sch“ geschrieben und einige Jahre später der gleiche Bauernhof mit „Hatschek“. Nichts entgeht seinem Auge! Oder, dass Verwaltungseinheiten generell nur auf Deutsch aufscheinen.

Im Zusammenhang mit den Vulgonamen hat Professor JoRDAN auch ein wunderschönes Wort erfunden, eine total nette Bezeichnung für entlegene Bauerndörfer, die in den topographischen Karten aufscheinen. Das Wort lautet: „Kleindenkmäler!“ Ist das nicht schön, wer denkt denn bei einer alten Keusche daran, dass diese ein Denkmal sei, ein Kleindenkmal. Danke Professor JoRDAN für diese liebevolle Wortschöpfung.

Eine andere schöne Redewendung lautet „verdichtete Erzählung“. Ein Ortsname ist eine Bezeichnung für eine Landschaftsstruktur, einen Teil eines Territoriums, ein Objekt, die auch emotional binden kann. Eine verdichtete Erzählung eben. Im Slowenischen „Rute“ zum Beispiel oder Podjuna (Jauntal), Košuta, Zila (Gailtal)!

Grundsätzlich meint Professor JoRDAN, dass Österreich nicht nur wegen rechtlicher und humanitärer Verpflichtungen, die sich aus dem Staatsvertrag ergeben, handeln soll, sondern auch im Interesse der Qualität und Aussagekraft seiner Staatskarten und - nicht zuletzt - zur Wahrung seines internationalen Ansehens. Er ist sich aber auch der Grenzen bewusst, denn Verbesserungen dieser Art müssen auch politisch durchsetzbar sein.

Erlauben Sie mir noch einige Gedanken, warum die Tätigkeit von Professor JoRDAN für die Volksgruppe so wichtig ist. Wie Sie wissen, sind die Karawanken sehr hoch und deshalb ist unser Horizont manchmal etwas eingeengt. Es ist deshalb ungemein wertvoll, wenn JoRDAN regelmäßig auch internationale Konferenzen besucht, wie zum Beispiel die 11. Konferenz der Vereinten Nationen zur Standardisierung geographischer Namen und die 30. Sitzung der Expertengruppe der Vereinten Nationen in New York, oder - erst unlängst - die erste neue Sitzung der Expertengruppe der Vereinten Nationen für geographische Namen, ebenfalls in New York. Er erhebt also unsere (kleine, provinzielle) Thematik (sicherlich nicht die einzige) auf ein ganz anderes Niveau, auf eine andere Ebene, zu der wir eigentlich keinen Zugang haben oder hatten. Ein Aspekt dieser Sitzung war auch die Transkribierung von topographischen Begriffen aus dem Arabischen in die lateinische Schrift. Auch das muss standardisiert werden.

Ein anderer Einspieler-Preisträger hat unsere Problematik ebenfalls auf Ebenen gehoben, wo die Volksgruppe keinen Zutritt hatte, zum Beispiel bei den Salzburger Festspielen. Ich spreche von der Uraufführung des Theaterstücks „Immer noch Sturm“ von Peter HANDKE. Ich denke auch an seine Übersetzungen von unseren großen Literaten LIPUŠ und JANUš. Es folgten dann Publikationen bei angesehenen Verlagshäusern. Solche Kontakte sind ungemein wertvoll und stellen für die Volksgruppe eine positive Expansion in andere Bereiche dar.

Ganz wesentlich aus der Sicht der Volksgruppe ist auch die Zusammenarbeit mit Institutionen der Volksgruppe. So fand auf Initiative von Professor JoRDan eine Konferenz der Arbeitsgruppe der Vereinten Nationen für Exonyme („UNGEGN Working Group on Exonyms“) in Tainach/Tinje statt, wo es 2010 um Toponyme und Exonyme ging und wo es angeblich auch zu leidenschaftlichen Auseinandersetzungen kam. Exonyme sind zum Beispiel Laibach, Pressburg oder Mailand für Ljubljana, Bratislava oder Milano. Vielleicht kann uns Herr Professor stichprobenartig etwas davon erzählen. Ich weiß auch nicht, wie man im Englischen sagt: Bleiburg oder Pliberk, Jauntal oder Podjuna? Gibt es da Regeln? 
Eine ungemein fruchtbare Zusammenarbeit entwickelte sich auch mit dem Slowenischen Volkskundeinstitut „Urban Jarnik/Slovenski narodopisni inštitut Urban Jarnik“ und dessen wissenschaftlicher Leiterin Martina PIKO-RUSTIA - und dies nicht nur, weil sowohl Urban JARNIK als auch Professor JORDAN im Gailtal geboren wurden. Diese Zusammenarbeit ist eine klassische Win-Win-Situation, wo beide Parteien von dieser Kooperation nur Vorteile haben. Und natürlich stellte Professor JoRDAN alle seine Kontakte zu Verfügung. Und umgekehrt.

Ich darf zum Abschluss kommen und abermals Professor JoRDAn zitieren. Durch die öffentlich gemachten Ortsnamen der Minderheit erfahren ihre Angehörigen ein Anerkennen und eine Wertschätzung ihrer Präsenz und ihres Anteils an der Identität eines Ortes und eines Landes durch die Mehrheitsgesellschaft. Umgekehrt bedeutet es aber ein Nicht-Anerkennen. Weiters unterstützen geographische Namen durch ihre emotional bindende Wirkung an die Heimat die Bindung der Minderheit an Orte und Landschaften, in denen sie leben. Das kann der Mehrheit doch nur recht sein. Die topographische Bezeichnung sagt aus: „Wir sind hier, wir sind präsent, wir wollen anerkannt werden!“ Also raumbezogene Identität!

Diese Sehnsucht kam auch im relativ kleinen Ort Köttmannsdorf/Kotmara vas zum Ausdruck, wo man davon ausgegangen ist, dass man von einer erstmals aufgelegten topographischen Karte mit Orts-, Flur- und Hausnamen 70 Exemplare verkaufen würde. Verkauft wurden aber laut Obmann Vinko Wieser 700 Karten. Praktisch jeder Haushalt hat also so eine Karte erworben. Hut ab! Und in Finkenstein/Bekštanj wurden bereits über 10.000 Exemplare der Karte mit slowenischen Flur- und Hofnamen aufgelegt. Diese werden vom dortigen Tourismusbüro, von der Gemeinde und vom slowenischen Kulturverein „SKD Jepa-Baško jezero“ sowohl den Einheimischen als auch den Touristen zur Verfügung gestellt. Das Interesse ist ungebrochen - auch in anderen Gemeinden.

Herr Professor JoRDAn befindet sich offiziell im Ruhestand, aber er ist der Auffassung, Ruhestand sei ungesund. Wir wissen, dass bei ihm viele Projekte im Gange sind, viele geplant sind und ebenso viele umgesetzt werden, treu seinem Rhythmus, eine Publikation pro Monat.

Zum Jubiläumsjahr 2020 wünscht sich Professor JoRDAN, dass man mehr als bisher betonen sollte, dass sich das, was Kärnten ausmacht, wesentlich aus zwei Quellen nährt. Erstens, dass sich die slowenische Volksgruppe nicht durch historische Zufälle in Kärnten befindet und die schon länger ansässige der beiden Volksgruppen ist und sie die gemeinsame Kärntner Kultur von Anfang an und zu wesentlichen Teilen gestaltet hat. Und zweitens wünscht sich Professor JoRDAN eine stärkere Präsenz dieser Kultur in der Namenlandschaft, in den Namen alpenslawischen Ursprungs, die in allen Teilen Kärntens vorkommen. Es bedeutet ein Anerkennen und Würdigen der Rolle der slowenischen Volksgruppe, wenn man ihre Namen öffentlich sichtbar macht, ob auf Ortstafeln oder in Karten. Welche schönen Wünsche zum 100. Jahrestag! Danke!

Wir wünschen Professor JORDAN bei seiner weiteren ungemein spannenden Tätigkeit viel Glück und Segen, viel Inspiration und neue Ideen und weiterhin eine gute Zusammenarbeit mit der Volksgruppe, ihren Institutionen, Mitarbeiterinnen und Mitarbeitern. Wir freuen uns darauf und danken bereits heute. Wir danken aber auch für die Freundschaft! Und gratulieren herzlich zum Einspieler-Preis!

\section{Verwendete Literatur}

Schwarz W., Kahl T. (2014): Peter Jordan - Ein bedeutender Kulturgeograph, Kartograph und Wissenschaftsmanager ist 65. In: Mitteilungen der Österreichischen Geographischen Gesellschaft, 156, S. 393-396.

JORDAN P. (1988): Möglichkeiten einer stärkeren Berücksichtigung slowenischer Ortsnamen in den heutigen amtlichen topographischen Karten Österreichs (= Österreichische Akademie der 
Wissenschaften, Institut für Kartographie, Berichte und Informationen, 6). Wien: Verlag der ÖAW.

JoRDAN P. (1992): Slowenische Ortsnamen in den amtlichen topographischen Karten Österreichs. Heutiger Zustand und Vorschläge zu seiner Verbesserung. In: Österreichische Namenforschung, 20 (1-2), S. 89-105.

JoRDAN P. (1999): Slowenische Ortsnamen in den amtlichen topographischen Karten Österreichs. Heutiger Zustand und Vorschläge zu seiner Verbesserung. In: Österreichische Namenforschung, 27 (1-2), S. 41-54.

Jordan P., Bergmann H., Burgess C., Cheetham C. (Hrsg.) (2011): Trends in Exonym Use, Proceedings of the $10^{\text {th }}$ UNGEGN Working Group on Exonyms Meeting, Tainach, 28-30 April 2010. Hamburg: Verlag Dr. Kovać.

JoRDAN P. (2012): Zur Bedeutung zweisprachiger geographischer Namen für die kulturelle Identität. In: Hren K., PANDel M. (Hrsg.): Ein Jahr danach. Die Ortstafelregelung 2011 und was daraus wurde $=$ Leto kasneje. Ureditev krajevnih napisov 2011 in kaj je iz tega nastalo. Klagenfurt / Laibach / Wien - Celovec / Ljubljana / Dunaj: Hermagoras / Mohorjeva, S. 125-146.

Jordan P. (2017): 11. Konferenz der Vereinten Nationen zur Standardisierung geographischer Namen und 30. Sitzung der Expertengruppe der Vereinten Nationen. In: Mitteilungen der Österreichischen Geographischen Gesellschaft, 159, S. 379-392.

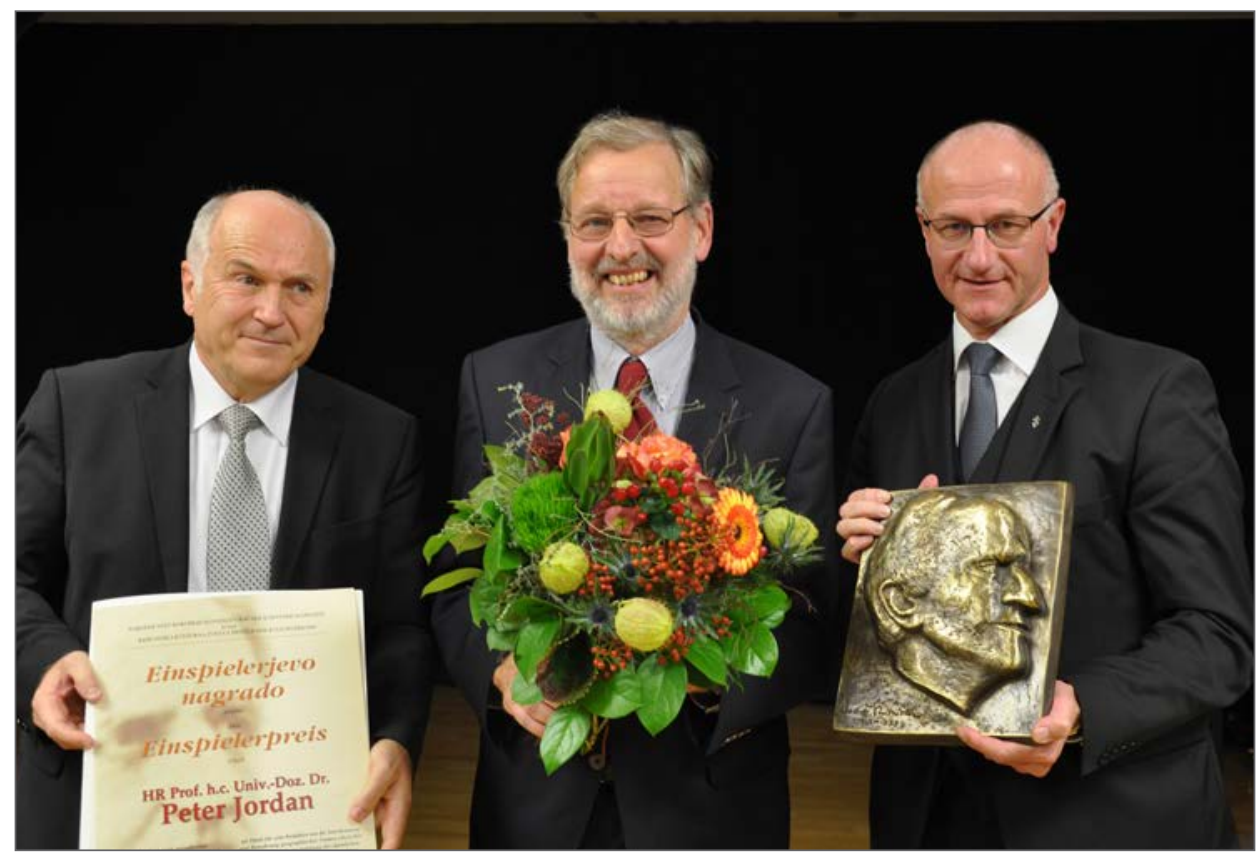

Foto: $\quad$ Milka OLIP, 2019

Abb. 1: Verleihung des Einspieler-Preises am 18. November 2019 in Klagenfurt an den Preisträger Peter JoRDAN (Bildmitte); links: Dr. Valentin InZKo, Vorsitzender des Rates der Kärntner Slowenen, mit der Urkunde; rechts: Mag. Janko KRIŠTOF, Vorsitzender des Christlichen Kulturverbandes, mit dem Bronzerelief von Andrej EINSPIELER 


\title{
Dankesworte von Peter Jordan
}

\author{
Preisträger, Einspieler-Preis 2019
}

Sehr geehrter Herr Botschafter, sehr geehrte Damen und Herren, liebe Freunde!

Ich freue mich außerordentlich über diesen Preis und bedanke mich sehr dafür. Ich habe es zunächst gar nicht glauben können. Als ich das Schreiben das erste Mal gelesen habe, habe ich gedacht, das wäre eine Einladung zu einer Preisverleihung an jemand anderen. Erst beim zweiten Lesen ist mir bewusst geworden, dass ich es bin, an den der Preis verliehen werden soll. Dann war ich auch sehr froh zu sehen, dass Heinz-Dieter PoHL den Preis schon bekommen hat. Denn, wenn ich den Preis bekäme und er ihn nicht schon bekommen hätte, wäre mir das als grob ungerecht erschienen.

Nach einigem Nachdenken war ich dann überwältigt und fast gerührt von der Dankbarkeit, die Sie mir damit entgegenbringen und dadurch ausdrücken - eine übergroße Dankbarkeit für etwas, das jeder andere anständige Mensch in meiner Position und mit meinen Möglichkeiten wohl auch getan hätte: sich für Minderheitenrechte einzusetzen, eigentlich auch nur für die längst fällige Durchsetzung bereits verbriefter Minderheitenrechte.

Eine weitere Relativierung: Ich neige prinzipiell dazu, spontan und ohne viel über die Folgen nachzudenken, für den jeweils Schwächeren Partei zu ergreifen. Und noch eine Relativierung: Ich trete für die Rechte von Minderheiten und indigenen Gruppen generell und überall in der Welt ein. Dazu gibt es öfter Gelegenheit im Rahmen der Vereinten Nationen, ihrer Expertengruppe für die Standardisierung geographischer Namen. Die Vereinten Nationen sind heute zwar sehr minderheitenfreundlich eingestellt und man muss dort nicht gegen den Strom schwimmen, wenn man sich für Minderheitenrechte einsetzt. Es gibt aber doch heikle Situationen, zum Beispiel, was die geographischen Namen der italienischen Minderheit in Südtirol betrifft, die sich heute ja in einer nicht-dominanten Position befindet. Deren Namen sind ja (mit Ausnahme derer, die es schon vor dem Ersten Weltkrieg gab) erst nach dem Ersten Weltkrieg von Ettore ToLOMEI erfunden oder aus dem Deutschen übersetzt worden. Sie sind aber mittlerweile die Namen einer Gruppe, die dort schon seit fünf oder sechs Generationen lebt. Und es sind die Namen, die sie mit ihrer dortigen Heimat verbinden. Man kann sie ihnen deshalb nicht wegnehmen.

Allerdings hat das alles wohl auch viel damit zu tun, dass ich aus Kärnten, aus Hermagor, komme, wo ich die ersten zwölf Lebensjahre verbracht habe und in die Volksschule und in zwei Klassen Hauptschule gegangen bin. Ich hatte schon in der Volksschule und noch mehr in der Hauptschule Schulfreunde aus dem gemischtsprachigen Gebiet. Sie waren damals noch deutlich an ihrer besonderen Aussprache des Deutschen zu erkennen. (Heute ist das ja nicht mehr so.) Mir wurde die Minderheitensituation damals also durchaus bewusst. Ich fand sie auch interessant und spannend, aber es wäre übertrieben, zu behaupten, dass ich mir schon tiefere Gedanken darüber gemacht hätte.

Das „Erweckungserlebnis“ war die Übersiedlung unserer Kernfamilie nach Salzburg, zunächst in den Flachgau und dann in die Stadt Salzburg. Eine Übersiedlung innerhalb Österreichs mag ja in reiferen Jahren keine so große Affäre sein. Wenn man als 12-Jähriger aus seinem Freundeskreis und seiner gewohnten Umgebung herausgerissen wird, ist das aber doch ein einschneidendes Erlebnis. Ich habe mich in eine andere Welt versetzt gefühlt. Und es wurde mir der Unterschied der Kulturen bewusst. Salzburg ist ja mit Ausnahme des Lungaus „urbairisch“ und die Unterschiede beziehen sich auf alle Kulturelemente: Die Sprache (der Dialekt) ist in Kärnten weicher und melodischer. Die traditionelle Baukultur ist in Kärnten mit den Walmdachhäusern, den Kesn oder den Marterln sehr typisch. Das Kärntner Liedgut ist etwas ganz Eigenes und Schönes - und deshalb freue ich mich auch sehr, dass das Karnitzen-Quintett heute hier singt. Das geht dann bis zur Namenlandschaft, 
die - wie Heinz-Dieter PoHL eindrucksvoll aufgezeigt hat - in ganz Kärnten, nicht nur im heute gemischtsprachigen Gebiet - eine völlig durchmischte ist.

Es wurde mir also bewusst, dass die Kärntner Kultur sich aus zwei Quellen speist, aus der alpenslawisch-slowenischen und aus der bairisch-deutschen, und dass es diese Mischung, diese glückliche Symbiose ist, die die Eigenart Kärntens und der Kärntner Kultur ausmacht. Das sollte man - gerade in einer Zeit, in der „regional branding“ sehr modern ist, auch viel stärker betonen. Das alpenslawisch-slowenische Element ist ein wesentlicher, konstitutiver Bestandteil der Kärntner Kultur.

Noch ein letzter Gedanke: Bei der letzten UNGEGN-Sitzung heuer im Frühjahr in New York wurde in einem Vortrag darüber berichtet, dass im nordöstlichen Baltikum (Estland, Leningrader Gebiet, Karelien) die Namen kleiner Minderheiten mit verklingenden und zum Teil schon verklungenen Sprachen im öffentlichen Raum (auf Ortstafeln und in der übrigen linguistischen Landschaft) wieder sichtbar werden. Das hat dort zur Folge, dass die Sprecher dieser Sprachen oder Menschen, die aus dieser Gruppe stammen, diese Namen anerkannt sehen und sich des Wertes dieser Namen und damit auch der Sprache bewusst werden - und sie wieder häufiger sprechen oder sogar neu erlernen. Das, so der Vortragende, sei dort Teil einer Art Jugendkultur. Das lässt auch für die Kärntner slowenischen Dialekte hoffen und zeigt, wie wichtig und verdienstvoll es ist, wenn Martina Piko-Rustia mit ihrem Team und Vinko Wieser slowenische Hof- und Flurnamen sammeln, dokumentieren und in Tourismus- und Wanderkarten publizieren.

Mit diesem optimistischen Ausblick möchte ich mich nochmals ganz herzlich bedanken. 